Recepción: 12/09/2018

Aceptación: 10/11/2018

Publicación: 20/01/2019

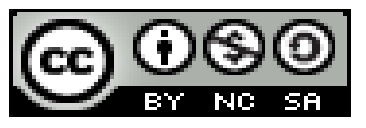

Ciencias técnicas y aplicadas Artículo de investigación

\title{
Incidencia del grado de humedad en suelos en textura franco limoso, ante la aplicación de las labores de arado y rastrado
}

\section{Incidence of the degree of humidity in soils with a loamy texture before the application of plowing and sculpting}

\section{Incidência do grau de umidade em solos com textura argilosa antes da aplicação de aragem e escultura}

\author{
Alfredo J. Carvajal-Rivadeneira ${ }^{\mathrm{I}}$ \\ ajcarvajal@utm.edu.ec \\ Richard X. Cevallos-Mera II \\ rcevallos@utm.edu.ec \\ Daniel D. Carvajal-Rivadeneira ${ }^{\text {III }}$ \\ daniel.carvajal@unesum.edu.ec \\ Emil C. Vega-Ponce IV \\ ecvega@utm.edu.ec
}

Correspondencia: ajcarvajal@utm.edu.ec

\footnotetext{
I Magíster en Pedagogía, Ingeniero Agrícola, Docente de la Facultad de Ingeniería Agrícola de la Universidad Técnica de Manabí, Portoviejo, Ecuador.

${ }^{\text {II }}$ Magíster en Educación y Desarrollo Social, Ingeniero Agrícola, Docente de la Facultad de Ingeniería Agrícola de la Universidad Técnica de Manabí, Portoviejo, Ecuador.

III Magíster en Administración Pública Mención Desarrollo Institucional, Ingeniero Civil, Docente de la Facultad de Ciencias Técnicas de la Universidad Estatal del Sur de Manabí, Jipijapa, Ecuador.

IV Doctor en Recursos Hídricos, Magíster en Ingeniería Agrícola con Mención en Recursos Hídricos, Ingeniero Agrícola, Docente de la Facultad de Ingeniería Agrícola de la Universidad Técnica de Manabí, Portoviejo, Ecuador.
} 


\section{Resumen}

El presente artículo tuvo como propósito estudiar la incidencia del grado de humedad en suelos de textura franco -limoso ante la aplicación de las labores de arado y rastrado. El estudio se realizó en la Finca Experimental de Riesgos del C.R.M. en el sitio Lodana del Cantón Santa Ana. El elemento básico que intervino fue el tractor en las labores de arado y rastrado en un suelo franco limoso con distintos grados de humedad, donde se tomó la resistencia que el tractor debe vencer para preparar el suelo, el tiempo empleado en las labores y la determinación del grado de pulverización del terreno a través del número y diámetro de los terrones existentes después de realizar las labores. Para ello, se utilizó un diseño experimental de bloques al azar con diez tratamientos y tres repeticiones. Los tratamientos presentaban distintos grados de humedad que variaron desde el 14,05\% la parcela más seca (testigo), hasta el 30,75\% la más húmeda (A9R9). Entre sus resultados, se destaca que es conveniente realizar las labores de arado y rastrado entre el $20 \%$ y el $27 \%$ de humedad, lo que permitió concluir que cuando se realizan las labores de arado y rastrado en un suelo seco, no queda convenientemente preparado, y en un suelo demasiado húmedo, se nota la compactación que sufre el suelo a más que este se adhiere a los implementos. En ambos casos se registraron las resistencias más altas.

Palabras clave: Grado de humedad; suelos de textura franco -limoso; labores de arado y labores de rastrado

\section{Abstract}

The purpose of the present study was to study the incidence of moisture content in soils with a clear texture when applied to plowing and drainage. The study was carried out in the Experimental Risks Farm of C.R.M. in the site Lodana of the Canton Santa Ana. The basic element that intervened was the tractor in the works of plow and astride in a loam franc soil with different degrees of humidity, where the resistance was taken that the tractor must overcome to prepare the soil, the time used in the work and the determination of the degree of spraying of the ground through the number and diameter of the clods existing after the work. For this, an experimental design of random blocks with ten treatments and three replications was used. The treatments had different degrees of humidity ranging from $14.05 \%$ to the dryest plot (control), to $30.75 \%$ wetter (A9R9). Among its results, it is worth noting that it is convenient to perform plowing and trailing between $20 \%$ and 
$27 \%$ moisture, which allowed to conclude that when plowing and trawling is carried out on a dry soil, it is not adequately prepared, and in a soil that is too moist, the soil compaction is noticeable, even though it adheres to the implements. In both cases, the highest resistances were recorded.

Key words: Degree of humidity; soils with a clear texture; plowing and plowing

\section{Resumo}

O presente artigo tem como objetivo estudar a incidência do grau de humedade em tecidos de franco -limor ante a aplicação dos laboratórios de arado e rastreado. O estúdio é realizado na Finca Experimental de Riesgos del C.R.M. en el sitio Lodana del Cantón Santa Ana. O elemento básico que intervém é o trator nos labores de arado e rastreado em um suelo franco limoso para as diferenças de humedade, é o mesmo que a resistência que o trator deve vencer para preparar o suelo, o tempo emplorado nos labores e a determinação del grado de pulverización del terreno a través del nuevo e diámetro de terrones aonde desppui de realizar labores. Para além disso, utilizase um esquema experimental de bloques e tratamentos repetitivos. Os tratamenhos presentaban distintos grados de humedecido que variam desde os 14,05\% a coleção mais seca (testigo), até os 30,75\% o mais húmeda (A9R9). Entre os resultados, veremos que é conveniente realizar os labores de arado e rastreio entre os $20 \%$ e os $27 \%$ de humedados, de modo a permitir que sejam concluídos os laboratórios de arado e rastreados em um suelo seco, sem queda conveniente, em um suelo demasiado húmedo, não é a compactação que sufre o suelo a mais que este se adere aos implementos. En todos casos registrador las resistencias altas.

Palabras clave: Grado de humedad; suelos de textura franco-limoso; labores de arado e labores de rastrado

\section{Introducción}

Actualmente, se vienen realizando continuas investigaciones sobre los diferentes parámetros relacionados con la eficiencia de las labores agrícolas, para lograr obtener las variables que más satisfagan los requerimientos de los agricultores, tendientes a obtener como finalidad el mejoramiento y el máximo rendimiento, tanto de su maquinaria, como en la utilidad económica, beneficio social y conservación ambiental. Para ello, se ha de considerar que la agricultura sostenible moderna, la constante y máxima eficiencia de la tecnificación agrícola, logran la optimización de los recursos disponibles y reducir los gastos y riesgos de producción, todo ello con los progresos obtenidos en la mecanización agrícola en los últimos años, para obtener mejores 
cosechas, reducir los residuos de cosecha, minimizar el costo de la mano de obra y preservar el medio ambiente (ecosistema).

Es de indicar, según Arnal y col (2006), que las labores agrícolas mecanizadas tienen como objetivo remover y preparar la tierra para mantenerla en buen estado y así obtener un resultado óptimo de cosecha, desde este punto de vista son muy importantes para la preparación y mejoramiento fisicoquímico del suelo, sin embargo, este efecto benéfico se nulita por su uso inadecuado y excesivo, o por las labores realizadas con un contenido de humedad del suelo inapropiada. Entre las causas y condiciones más comunes que dificultan las labores agrícolas se puedenmencionar:

- La consistencia del suelo debido a diferentes porcentajes de humedad, que está relacionada con el contenido de humedad óptimo para efectuar la labranza.

- La presencia de suelos pesados debido a su textura arcillosa que varía el grado de penetración que ofrece el suelo a los diferentes aperos de labranza.

- La presencia de suelos compactos, como consecuencia del uso inadecuado y excesivo de la mecanización agrícola en la explotación agropecuaria, por la presencia de ganado, a la falla de técnicas para conservar el suelo, etc.

- La presencia de material orgánico e inorgánico como troncos, raíces, material pétreo y otros elementos extraños que se encuentran en el suelo y que dificultan la labor de labranza del mismo.

- El grado de pendiente del terreno, a mayor pendiente, mayor dificultad.

En atención a las causas expuestas y de acuerdo con las características de los suelos manabitas en general, es importante tener en cuenta el contenido de materia orgánica. La materia orgánica tiene una alta capacidad de absorción de agua, su hidratación alarga la disponibilidad de agua para la formación de las películas alrededor de las partículas del mineral. La presencia de materia orgánica hace posible la labranza con 52,2 \% de humedad sin enfangar el suelo. Si se quita la materia orgánica, basta un $27.7 \%$ de humedad para que las labores de cultivo conviertan el suelo en un barrizal. La falta del recurso hídrico suministrado en forma natural o mediante el riego artificial, la escasa economía, la poca tecnología aplicada por nuestro agricultor en las producciones agrícolas, un manejo inadecuado de los suelos manabitas los hacen pobres en materia orgánica al suelo para permitir que las labores de labranza se realicen de la forma más óptima tomando en cuenta la humedad existente en el suelo; la edición de materia orgánica a los suelos extiende la zona de friabilidad a contenidos de humedad bastante elevados. Además, se hace necesario recalcar el 
manejo óptimo que se debe dar a los diferentes aperos de trabajo que participan en la mecanización agrícola. Para ello es importante considerar su material de construcción, la adecuada regulación de los implementos según la condición actual del suelo, así como el empleo de operadores capacitados para el manejo, funcionamiento y mantenimiento de las maquinarias agrícolas. Ante tal planteamiento, en el presente artículo se determinó la incidencia del grado de humedad en suelos de textura franco -limoso ante la aplicación de las labores de arado y rastrado.

\section{Desarrollo}

\section{La incidencia del agua en la preparación del Suelo.}

En la actualidad existe un buen número de dispositivos para determinar el contenido en agua del suelo basado en técnicas muy diferentes. De forma general según Fernández (2005), estas técnicas pueden agruparse en cinco grupos: gravimétricas, tensiométricas, atenuación de neutrones, disipación de calor y técnicas dieléctricas. Por otro lado, destaca el hecho en la cual explica que la medición directa del contenido en agua del suelo se realiza mediante pesada y secado de un volumen de suelo conocido, pero este tipo de determinaciones, además de laboriosa, es destructiva $\mathrm{y}$, por tanto, no adecuada en muchos casos. Al ser la única medida directa, aunque no exenta de errores, es la metodología de referencia y base para la calibración del resto de técnicas. Otras técnicas se basan en medidas indirectas de propiedades del suelo que varían a su vez con el contenido en agua. Ahora, algunas de estas nuevas técnicas están siendo ampliamente utilizadas en regiones áridas y semiáridas, alcanzando un nivel de automatización elevado, lo que permite disponer de información detallada imprescindible para entender el comportamiento del agua en el suelo.

Según Gavande, S (1991), la preparación o labranza del terreno tiene relación a las diversas manipulaciones mecánicas que mantengan a los suelos en óptimas condiciones para el desarrollo de los cultivos, siendo importante en el efecto benéfico o perjudicial de dicha labranza el tipo de implementos empleados y de la intensidad de su uso. Entre los propósitos que persigue la preparación de los suelos mediante la mecanización tenemos los siguientes:

- Proporcionar un lugar adecuado para que la semilla germine y para que se desarrollen convenientemente las raíces de las plantas. De esta manera se garantiza una buena formación de materia orgánica en el suelo. 
- Destruye y controla las malas hierbas en el momento de la preparación del terreno, la estresada y brinda a su vez mayor incorporación de materia orgánica al suelo.

- Conservar o mejorar la estructura del suelo, brindando al lote la posibilidad de una mejor y mayor aireación y evitando la formación de costras en la superficie.

- $\quad$ Prepara el terreno de tal modo que pueda penetrar en el suelo la mayor cantidad posible de agua, en lugar de escurrimiento por la superficie y producir erosión. Es conveniente por varias razones, una preparación del terreno que permita que penetre el agua de lluvia rápidamente, un escurrimiento menor significa también menos erosión.

Todos estos propósitos se consiguen, según Ribes y col (2005), con varios factores que deben estar presentes en el suelo al momento de realizar la labor agrícola. Es así, que la humedad más conveniente para la labranza varía considerablemente. La labranza del suelo con humedades inferiores a la óptima tiende a destruir la estructura, y cuando están muy mojados se puede convertir en lodo. Es por esta razón, que, con un bajo contenido de humedad, el suelo es muy duro y muy coherente por el efecto cementante entre las partículas secas; el suelo se aterrona si es arado en estas condiciones.

Al aumentar el contenido de humedad el agua es absorbida por las partículas del suelo, debilitando la coherencia y comunicando friabilidad. La zona de consistencia poco resistente representa el intervalo de humedad del suelo, en el cual las condiciones de labranza son óptimas. Es decir que las fuerzas que mantienen unido el material que forma los gránulos del suelo se debilitan cuando este está húmedo. De ahí, tal como plantea Castilla (2013), se puedan labrar, preparar y cultivar estos suelos cuando están ligeramente húmedos, pero no muy húmedos porque la máquina puede a lascarse y no queda convenientemente preparado para la siembra, produciéndose la compactación en las áreas donde se realizan las vueltas de las labores de arado y rastra por los pases de los neumáticos. Si se labran y gradean los suelos pesados cuando contienen la cantidad adecuada de humedad, estas operaciones contribuyen eficazmente a la formación de gránulos. Según investigaciones realizadas, el contenido óptimo de humedad del terreno para las labores varía alrededor del $40 \%$ al $50 \%$ de humedad.

Además, hay que considerar la compactación del suelo, que es una consecuencia negativa de la inapropiada y excesiva mecanización del terreno. Es así, que los mecanismos de rodadura de los tractores al estar en contacto con el suelo ejercen una presión sobre este. Dicha presión produce un 
aplastamiento en los suelos blandos que disminuye el número de poros, tomándose mayor este efecto cuando más húmedo está el terreno.

\section{El agua en las propiedades dinámicas de los suelos.}

Las propiedades dinámicas de los suelos se manifiestan en los movimientos que resultan de las fuerzas externas aplicadas y es así como Gill y Van denBerg (1998), definen a la dinámica de los suelos como "la relación entre las fuerzas aplicadas al suelo y la reacción resultante del mismo" (p.23).

"Los objetivos de la labranza solo se alcanzan mediante la aplicación de fuerzas a los suelos con los aperos y máquinas agrícolas" (p.45). Por otro lado, Bourennane y col (2004), explica que cuando una determinada cualidad del suelo se relaciona con muchas de sus propiedades, se deben seleccionar aquellas más relevantes. Técnicas geoestadísticas multivariadas pueden suministrar medidas cuantitativas de interacciones complejas entre propiedades del suelo, y son particularmente útiles para formular hipótesis sobre qué causa específicamente la variabilidad.

Por otro lado, se destaca lo propuesto por Guastaferro y col. (2010), quienes utilizaron el análisis de componentes principales para reducir el número de variables a tener en cuenta para definir zonas de manejo en programas de agricultura de precisión. Y Chen y col (2004) utilizaron el análisis de componentes principales para derivar información específica de fotografías aéreas a color que les permitió eliminar la interferencia que producía la materia orgánica en el color de la superficie del suelo desnudo y pudieron hacer un mapa de la distribución superficial de arcilla en un campo de 115 hectáreas.

Por su parte, Zagal (2007), el valor de la cortadura o coeficiente de resistencia interna de los suelos, que se la considera como la resistencia al deslizamiento o resbalamiento del suelo sobre el suelo, es generalmente aceptado como la principal propiedad dinámica en las interacciones entre el suelo y la maquinaria. Por otro lado, la fricción entre el suelo y el metal es una variable importante en las operaciones de labranza. Nichols (1929), observó que existen tres fases en el razonamiento entre el suelo y el metal, las cuales dependen del contenido de humedad, del peso y material de la herramienta de metal. Por su parte es importante señalar, que la friabilidad es la facilidad que tiene el suelo de desmenuzarse, en la que juega un papel importante la humedad existente en el mismo. El estado de humedad en que los suelos son friables es el de los mejores para la labranza. Los suelos mullidos y friales son más recomendados de trabajar, ya que estos suelos son los que poseen mejor estado de agregación (mejor textura) para el cultivo. 
En cuanto a la plasticidad de un suelo, se ha demostrado que estos (excepción de los que carecen de plasticidad, como las arenas) se hacen más plásticos a medida que aumenta el contenido de humedad. La plasticidad se la ha definido como; la propiedad que le permite a la arcilla cambiar su forma sin romperse cuando se la somete a un esfuerzo de deformación, o la propiedad que permite la deformación sin ruptura cuando el material es sometido a una fuerza que excede el límite de elasticidad. A la plasticidad se la considera como una característica de la arcilla que le permite captar agua y construir una masa que pueda adquirir cualquier forma aumentando su volumen y que conserva esta forma al cesar la presión de deformación. El laboreo del suelo en estado plástico o con una consistencia dura provoca siempre condiciones desfavorables, ya que, a bajos contenidos de humedad, las partículas no se unen y, por consiguiente, no forman agregados, y a altos contenidos de humedad al secarse el suelo producen terrones grandes y compactos. El suelo debe manipularse a un contenido de humedad de consistencia friable.

Según investigaciones realizadas, el valor de cortadura o resistencia al esfuerzo cortante aumenta hasta alcanzar un máximo con el contenido de humedad del límite plástico, decreciendo luego bruscamente hasta un valor bajo en el límite líquido. El valor máximo es proporcional al índice de plasticidad. Casi todas las variables del suelo antes citadas contribuyen al valor de la componente del esfuerzo cortante. En cuanto a la consistencia del suelo, es indispensable conocer que esta comprende todas las manifestaciones de las fuerzas físicas de cohesión y adhesión que obran dentro del suelo a diferentes constantes de humedad. La fuerza de cohesión está en función de los coloides de la tierra y de su humedad. Es por esta razón, que el agua juega un papel importante en el valor de la cohesión, pues afecta a las distancias entre las partículas y las fuerzas de atracción asociadas con los meniscos aire-agua. La fuerza de adhesión se refiere a la atracción de la fase líquida sobre la superficie de la fase sólida.

Las moléculas de agua pueden adherirse a las partículas del suelo a los objetos puestos en contacto, por ejemplo, los aperos de labranza. La cohesión en los suelos es en cambio, la unión entre partículas debido a las fuerzas de atracción mutua que surgen de mecanismos físicos-químicos. Es por esta razón que, en mayor aumento de la cantidad de agua, la cohesión de las películas de agua alrededor de las partículas hace que el suelo se vuelva plástico.

En cuanto a las manifestaciones de las fuerzas de cohesión y adhesión para la consistencia de un suelo tenemos:

- El comportamiento ante la gravedad, la presión, el empuje y la tracción. 
- La tendencia de la masa del suelo para adherirse a cuerpos extraños, como los implementos de labranza.

- Las sensaciones del tacto en los dedos del observador.

- Según esta definición, el concepto de consistencia del suelo incluye la resistencia a la compresión, resistencia al esfuerzo cortante, friabilidad, plasticidad y glutinosidad, propiedades que se manifiestan de modo diferente según varían la fuerza de cohesión y de adhesión en la masa del suelo.

\section{Dinamómetros}

Nichols (1929), atacó el problema al medir la potencia necesaria para que el arado pueda vencer las fuerzas de cohesión, adhesión, la resistencia al esfuerzo cortante, y la fricción entre el suelo y el metal, jalando cinceles a través del suelo. Los suelos fueron compactados con diversos "contenidos de humedad" a cada cincel se le acopló un pequeño dinamómetro para registrar la fuerza de tracción en libras. Es de indicar, según Buduba (2004), que el contenido de humedad del suelo es el factor más importante que contribuye a la fricción entre el suelo y el metal. Un análisis del efecto humedad sobre la resistencia del peso de los cinceles mostró que la máxima tracción ocurría en el intervalo plástico, algo por encima del límite plástico. Como los factores primarios que afectan la resistencia del suelo al paso de los implementos de labranza son la cohesión y adhesión es evidente que la resistencia máxima deba ocurrir en el intervalo de plasticidad. La tracción máxima aumenta en grado logarítmico en función del índice de plasticidad.

El tiro de arado en condiciones normales puede medirse con dinamómetros especiales. El ejemplo más notable de estudio con dinamómetros es el trabajo de Keen y Maíz (1925), realizado en la estación experimental de Rothanested (2015). Ellos perfeccionaron la uniformidad del suelo en relación con el tiro y el efecto de los tratamientos del suelo en el tiro del dinamómetro. En su análisis de las fuerzas de tiro en un campo, unieron en una gráfica los puntos de igual intensidad de tracción y formaron curvas de igual tiro con dinamómetros como un plano topográfico contiene curvas del mismo nivel.

En sus ensayos, Rothanested (2015), observó que la resistencia máxima fue 40\% más alta que la resistencia más baja registrada. Por otro lado, las raíces vivas de la vegetación juegan un papel importante en variar la tracción dinamométrica durante la operación de arar. El corte o aflojamiento de las numerosas raíces que existen en la capa arable consume trabajo. Es indispensable señalar 
además que los distintos órganos de una maquinaria o de una transmisión de movimiento se encuentran sometidos a una serie de esfuerzos que tratan de deformarlos o romperlos. Por esto la mecánica agrícola, ha entrado hace años al campo de las investigaciones experimentales. Gracias a las valiosas contribuciones de los técnicos que a ella se dedican, se hayan hoy empeñados de encarar en forma racional estos estudios, con evidente beneficio tanto para el fabricante de máquinas como para el mismo agricultor que podrá, según el caso, conocer las características de las máquinas que mejor respondan a sus necesidades.

Los constructores que estudien técnicamente una maquina calculan la forma y tamaño de cada uno de estos órganos de acuerdo con principios racionales de estática y dinámica. Así el estudio de una máquina agrícola abarca aspectos distintos que son:

- Su examen orgánico, que nos permitirá deducir la racionalidad de su construcción, materiales, empleados, etc.

- Su ensayo dinámico, para apreciar la fuerza necesaria para poder accionarla, la repartición de esta entre los distintos órganos y el rendimiento mecánico del conjunto.

- Su aplicación haciendo de ella el estudio agronómico y económico sobre el terreno, ocomo la maquina fija.

- De esta manera, podrá apreciar la perfección del trabajo realizado, la cantidad de este, su costo, la modalidad del manejo, conservación, etc. de la máquina en examen. El ensayo dinámico como objetivo de la experimentación de la Mecánica Agrícola, tiene como propósito establecer las fuerzas a que están sometidos los aperos, y los efectos siguientes:

- Tracción, cuando la pieza está sometida a la acción de dos fuerzas antagonistas que tratan de alargarla.

- Compresión, cuando la pieza se encuentran sometida a la acción de dos fuerzas antagonistas que tratan de acotarla.

- Flexión, cuando las fuerzas actúan tratando de curvar la pieza.

- Torsión, cuando las fuerzas actúan en forma de hacer girar la pieza alrededor de su propio eje, manteniendo un extremo fijo.

Para el ensayo dinámico de las máquinas se emplean aparatos que, con el nombre genérico de dinamómetros nos permiten determinar la intensidad de la fuerza para accionarlas en sus diferentes 
formas y a manifestaciones. Existen diferentes tipos de dinamómetros: los de tracción, de rotación, de oscilación, de compresión, de absorción o frenos dinamométricos.

Los dinamómetros de tracción tienen como principio general sobre el cual descansa la construcción de todos dinamómetros, el de dispositivos que permiten medir la deformación que sufre un resorte o muelle de acero sometido a la acción de un peso o fuerza. La deformación de estos órganos aumenta, aunque no en forma absoluta, en proporción a la fuerza que actúa sobre ellos. Las balanzas de resortes o romanas constituyen una aplicación de este principio; aumentando el peso, es decir la fuerza que actúa sobre el resorte, el índice corre sobre la escala graduada y nos indica la intensidad del esfuerzo, expresado en kilogramos. Toda vez que el esfuerzo desarrollado es variable, la aguja oscila y se desplaza de un punto a otro; debe entonces apreciarse un término medio del esfuerzo a criterio del experimentador y esto puede inducir a errores sensibles.

El dinamómetro Dina-enlace M.S.I representa un avance sobre intentos electrónicos anteriores para reemplazar los dinamómetros mecánicos. Los modelos Dina-enlace de la serie ofrecen características estándares que no se encuentren en otros dinamómetros. Este tipo de dinamómetro se caracteriza por ser de construcción impermeable, en su pantalla de cristal líquido están claramente anunciadas todas las funciones y unidades. Además, indican las fuerzas de tracción en varias unidades de medidas como libra, toneladas, toneladas métricas y de Ka Newtons.

\section{Rendimiento y capacidad de trabajo de las máquinas agrícolas}

Para Hiel, (2006), las exigencias en las prestaciones de las maquinarias están directamente relacionadas con el nivel de complejidad que exponen los equipos, siendo los autopropulsados (tractores y cosechadoras) los de mayor contenido tecnológico, así como las sembradoras para siembra directa tanto de grano fino como grueso. Asimismo, plantea Putt y col (2012), que el laboreo excesivo en los cultivos agrícolas, el riego excesivo, la eliminación de la cobertura vegetal, y el arado en tierras marginales afectan en gran medida el terreno. Por esta razón, plantean Mehuys y col (2009), que es necesario la innovación en nuevas técnicas de cultivo que ayuden a mitigar la erosión en los suelos e incrementar la capacidad de infiltración, permitiendo la optimización del agua, evitar la compactación y mantener los nutrientes del suelo en estado favorable para la siembra.

El rendimiento de la máquina en la mayoría de las veces será menor al 100\% ya que esta no trabaja al máximo de su capacidad por varios factores como la pérdida de tiempo al dar vueltas, para hacer ajustes en el tractor e implementos, en reparaciones pequeñas, la falla de continuidad del operador 
al efectuar su trabajo, etc. Además, este rendimiento de la maquina está afectado por otros factores como son el tipo de máquina, las distancias recorridas durante el trabajo, la potencia disponible y las condiciones del terreno ente las cuales tenemos el grado de humedad existente en el suelo, la pendiente, el porcentaje de materia orgánica, la textura y estructura del suelo, su plasticidad, su cohesión, adhesión, su consistencia, etc. Este rendimiento efectivo de campo se calcula dividiendo el área del trabajo realizado por el tiempo que se empleó en labrar dicha extensión de terreno.

La capacidad efectiva de labranza del suelo depende de factores como el ancho de trabajo de la máquina, la capacidad máxima de producción de la máquina, de la capacidad promedio, de la velocidad de avance y los tiempos perdidos durante la ejecución del trabajo. En cuanto a la velocidad de avance de la máquina, está es influenciada por:

- La fuerza de tiro necesaria del tractor para halar el implemento y así vencer las fuerzas de cohesión, adhesión, compresión, al esfuerzo cortante, la plasticidad, la friabilidad, la fricción entre el suelo y el metal entre otras, donde es influyente la humedad que existe en el suelo al momento de realizar la labor agrícola.

- Las propiedades del suelo como la textura, humedad, materia orgánica, etc., pueden limitar la velocidad de la maquinaria.

- La potencia disponible en la barra de tiro o en el enganche de tres puntos.

- Variaciones en la pendiente del terreno y la resistencia a la rodadura.

- Limitaciones como la altitud (m.s.m.m) al desarrollo de la potencia máxima.

\section{Materiales y métodos}

El presente trabajo se llevó a cabo en el valle medio del río Portoviejo, en la finca de experimentación de riesgos, que pertenece al M.A.G, y que actualmente se maneja por medio de un convenio cuatripartito en el que participan el Ministerio de Agricultura y Ganadería (M.A.G) el centro de rehabilitación de Manabí (C.R.M) el instituto Nacional de investigaciones Agropecuarias (I.N.I.A.P) y la Facultad de Ingeniería Agrícola de la Universidad Técnica de Manabí. El clima presenta: una temperatura media de $25,1^{\circ} \mathrm{C}$, precipitación de $543 \mathrm{~mm}$, humedad relativa de $78 \%$, horas sol media mensual de 112,9 horas y décimas y nubosidad de 8 octavos. En cuanto al análisis de suelo una textura franco limoso con un 22\% de arena, 59\% de limo, 19\% de arcilla y capacidad de campo de $50 \%$. 
En cuanto a las maquinarias y accesorios agrícolas utilizadas, se contó con un tractor de una potencia máxima de $2410 \mathrm{r} / \mathrm{min}$ y un régimen de ralentí de 550 - $650 \mathrm{r} / \mathrm{min}$; también con un arado con tres Vertederas y un ancho de corte de 1,20m de estructura rectangular y con un montaje semi - integral. Asimismo, se utilizó una Rastra con 22 discos de $60 \mathrm{~cm}$ de diámetro de diseño excéntrica y con un montaje semi - integral. También se contó con un Dinamómetro, Sistema de riesgo y el equipo para el análisis de suelo (Estufa, Cápsula, Balanza analítica de precisión, Agitadores de vidrio), entre otros accesorios se utilizaron cinta de 30 metros, jalones, cronómetro, machete y fundas.

Asimismo, se consideró durante la investigación los siguientes factores:

- La resistencia que ofrece el suelo a las labores de arado y rastrado, según el grado de humedad presente en el suelo al momento de las citadas labores en un suelo de textura franco limoso.

- El tiempo empleado en las labores primarias, así como el tiempo efectivo de trabajo.

- La eficacia de la labranza observando la pulverización del terreno.

La metodología siguió un diseño experimental con bloques al azar, en la cual se realizaron 10 tratamientos con 3 repeticiones a un número de 30 parcelas. Para el tratamiento se utilizó una parcela testigo (T), arada y rastrada con la humedad natural existente en el suelo y 9 parcelas arada y rastradas; una después de 3 días con mayor grado de humedad (A9R9) y el resto después de 6 (A8R8), 9(A7R7), 12(A6R6) ,15(A5R5), 18(A4R4), 21(A3R3), 24(A2R2) y 27(A1R1) días de haber llevado el suelo a capacidad de campo. Es de indicar, que antes de realizar las citadas labores, se tomaron muestras, para luego en el laboratorio de la facultad de Ingeniería Agrícola realizar los análisis correspondientes, para determinar el grado de humedad existente en el suelo al momento de realizar las labores. Es importante señalar que las labores en la parcela testigo se realizaron al inicio de la investigación.

Para el análisis del grado de humedad del suelo, se utilizó el método de la estufa a $105^{\circ} \mathrm{C}$, a través del siguiente cálculo

$$
\% \text { Humedad }=\frac{\text { Peso tierra húmeda }- \text { peso de tierra seca }}{\text { Peso tierra húmeda }} * 100
$$

Para conocer la Fuerza de tracción, se procedió a arar el terreno con el implemento montado sobre la unidad, ajustándolo en sentido horizontal y vertical. Cuando se efectuó la operación de aradura, se determinó la resistencia que ejercía el suelo al momento de la labor en cada parcela con grado de humedad diferente, con el dinamómetro instalado para el efecto y la evaluación la capacidad 
efectiva de trabajo, se realizó la operación de aradura en cada parcela utilizando el tractor y el respectico implemento. La capacidad efectiva de trabajo es el tiempo necesario para arar una parcela dependiendo del tiempo productivo y el tiempo improductivo (tiempo perdido en dar vuelta en las cabeceras). En cuanto al grado de pulverización del terreno, se contaron los números de terrones existentes en el suelo después de la labor y el diámetro de estos en tres muestras de $1 \mathrm{~m}^{2}$ cada una en todas las parcelas.

La labor del rastrado para la Fuerza de tracción incluyo la rastra montada en el tractor para ejecutar la operación. Su capacidad efectiva de trabajo incluyo la labor de gradeo y la obtención del grado de pulverización del terreno posterior a la realización de la labor de rastrado, se procedió por igual durante la labor de arado.

Para el análisis estadístico, se utilizó el Análisis de Variancia (ADEVA o ANAVA) y que según González (2004), es un procedimiento aritmético que consiste en desdoblar una suma de cuadrados total (variación total), en fuente de variación reconocida, incluyendo además la variación que no se ha podido medir, fuente de error que se conoce como residuo, resto o error experimental (proveniente de la variabilidad inherente al material experimental o de la falta de homogeneidad del ambiente en el que se realizó el experimento).

\section{Resultados}

Los resultados se establecieron con las lecturas de las residencias que el suelo ofrecía a las labores de arado y rastrado, al tiempo empleado en las labores citadas, así como en el grado de disgregación del suelo analizado a través del diámetro y número de terrones. Para el análisis estadístico solo se consideró como variable el grado de humedad existente en el suelo al momento de realizar las labores de arado y rastrado en cada uno de los tratamientos. Los grados de humedad obtenidos al momento de realizar las labores variaron desde el 14,05\% hasta el 30,75\%.

\section{Resistencia del suelo al arado.}

Los valores obtenidos en la resistencia del suelo franco limoso al arado, presenta que la resistencia más baja que se obtuvo con el ajuste de la curva fue de $333 \mathrm{Kg}$ a 23,5\% de humedad, mientras que la máxima resistencia registrada fue de $528,57 \mathrm{Kg}$ en el tratamiento testigo a $14,05 \%$ de humedad. El porcentaje entre ambas resistencias disminuye en un $37 \%$ (de 528,57 Kg a $333 \mathrm{Kg}$ ) en 9,45\% de diferencia de grado de humedad existente en el suelo (de 14,05\% a 23,5\%). 
Mediante el método de ajuste de la curva se estableció el grado de humedad óptimo para realizar la labor de arado. Dicho grado de humedad está en el 23,5\% donde la resistencia es de 333Kg. para la maquinaria y las condiciones establecidas.

El Factor Calculado (F.C.) obtenido mediante el análisis de la variancia es, con relación a los bloques significativos, y con relación a los tratamientos altamente significativo.

\section{Resistencia del suelo al rastrado.}

En los valores obtenidos en la resistencia que el suelo ofrece a la labor de rastrado, presenta que la resistencia más baja registrada se dio en el tratamiento A7R7 a 27,98\% de humedad y esta fue de $401,24 \mathrm{Kg}$, mientras la máxima resistencia registrada fue de $531,33 \mathrm{Kg}$ en el tratamiento testigo. El porcentaje entre ambas resistencias disminuye en un $24,48 \%$ (de $531,33 \mathrm{Kg}$ a $401,24 \mathrm{Kg}$ ) en $13,93 \%$ de diferencia de humedad existente en el suelo (de 14,05\% a 27,98\%). El F.C. obtenido mediante el análisis de la variancia es, con relación a los bloques bajamente significativos y con relación a los tratamientos altamente significativo.

\section{Tiempo empleado en la labor de arado.}

Según los datos obtenidos en el tiempo que se empleó en cada pase de la labor de arado, presenta que el tiempo más bajo registrado se dio en el tratamiento A4R4 donde se empleó un promedio de 56,44 segundos en cada pase y el mayor tiempo se registró en el tratamiento A3R3 donde se emplearon 75 segundos en cada pase.

El porcentaje entre ambos tiempos disminuye en un 24,75\% (de 75 ser. A5644 ser) en 11,94\% de diferencia de humedad existente en el suelo (de 18,65\% a 30,59\%). El F.C. obtenido mediante al ANAVA con relación a los bloques resultó significativo y con relación a los tratamientos significativos.

\section{Tiempo empleado en la labor de rastrado}

Los datos obtenidos en el tiempo empleado en la labor de rastrado presentaron que el tiempo más bajo registrado fue de 37,67 segundos en el tratamiento A6R6 y el tiempo más alto registrado fue de 51,67 segundos en el tratamiento A1R1 empleado en cada pase.

El porcentaje entre ambos tiempos disminuye en un 27,10\% (de 51,67 ser a 37,67 ser) en 10,74\% de diferencia de humedad existente en el suelo (de 14,54\% a 25,28\%).

El F.C. obtenido mediante el ANAVA es, con relación a los bloques bajamente significativo y con relación a los tratamientos resultó significativo. 


\section{Diámetro de terrones en la labor de arado}

Los datos obtenidos en el diámetro de terrones en la labor de arado, presenta que en el tratamiento A9R9 se registró el diámetro de terrones más bajos y este fue de $12,58 \mathrm{~cm}$, mientras en el tratamiento A1R1 se registró el diámetro de terrones más elevado y fue de $15,73 \mathrm{~cm}$.

El porcentaje entre ambos diámetros disminuye en un $20,03 \%$ (de $15,73 \mathrm{~cm}$ a $12,58 \mathrm{~cm}$ ) en $16,70 \%$ de diferencia de humedad existente en el suelo (de $14,05 \%$ a 30,75\%).

El F.C obtenido en el ANAVA es, con relación a los bloques bajamente significativos y con respecto a los tratamientos significativo.

\section{Diámetro de terrones en la labor de rastrado}

Los datos obtenidos en el diámetro de terrones en la labor de rastrado, presenta que el tratamiento con menor diámetro de terrones fue el A8R8 con 11,51 cm y en el tratamiento que mayor diámetro de terrones fue el A3R3 con un promedio de 13,23 cm por terrón.

El porcentaje entre ambos diámetros disminuye en un 13\% (de 13,23 cm a 11,51cm) en 11,94\% de diferencia de humedad existente en el suelo (de 18,65\% a 30,59\%).

El F.C. tanto en los bloques como en los tratamientos resultó que fue bajamente significativo.

Se compara la distribución de los diámetros de terrones en la labor de arado y rastrado en los diferentes tratamientos.

\section{Número de terrones en la labor de arado.}

Los valores obtenidos en el número de terrones en la labor de arado, presenta que el menor número de terrones que observó en el tratamiento A8R8 con 7,67 unidades por m2, y el mayor número de terrones se registró en el tratamiento testigo con 18,67 unidades por m2.

El porcentaje entre ambos números de terrones disminuye en un 52,92\% de 18,67u, a 7,67u.) En $16,54 \%$ de diferencia de humedad existente en el suelo (de 14, 05\% a 30,59\%).

El F.C. obtenido mediante el Análisis de Variancia es, con relación a los bloques bajamente significativo y con relación a los tratamientos significativos.

\section{Número de terrones en la labor de rastrado.}

Los valores obtenidos en el número de terrones, presenta que en el tratamiento A3R3 se registrará el menor número de terrones con 6,33 unidades por $\mathrm{m} 2$ y el tratamiento testigo con 17,67 unidades por $\mathrm{m} 2$ fue el que mayor número de terrones registro.

El porcentaje entre ambos números de terrones disminuye en un 64,17\% (de 17,67 u. a 6,33 u.) en $4,60 \%$ de diferencia de humedad existente en el suelo (de 14,05\% a 18,65\%). 
El F.C. obtenido mediante el Análisis de Variancia es, con relación a los bloques significativo y con relación a los tratamientos altamente significativo.

\section{Discusión.}

En la labor de arado, la humedad de suelo juega un papel importante en cuanto a la resistencia que ofrece el suelo en el momento de ejecutarla, es así como la diferencia que hubo entre la máxima resistencia y la mínima resistencia fue de 195,57 Kg, es decir de 37 \%. Este porcentaje es casi igual al que Rothansted (2015), observo en sus ensayos. Dicho porcentaje fue del $40 \%$.

La curva de resistencia del suelo franco limoso con distintos grados de humedad en la labor de arado tiene tendencia parabólica. El tratamiento testigo es el que menor porcentaje de humedad presentaba $(14,05 \%)$, se obtuvo la mayor resistencia, fue bajando paulatinamente hasta el tratamiento A6R6 donde se presentó la más baja resistencia. Luego la resistencia aumento hasta el tratamiento A9R9 (parcela con 30,75\% de humedad) donde se registró la segunda resistencia más elevada, esto como consecuencia de que el suelo contenía agua y lo volvía más pesado. Cabe indicar que en los dos últimos tratamientos (A8R8 y A9R9) que tenían un porcentaje de humedad de $30,59 \%$ y $30,75 \%$ respectivamente, el suelo se adhiere a las rejas del arado y los neumáticos de tractor producían una compactación que se veía a simple vista.

En cuanto a la resistencia que un suelo franco limoso con distintos grados de humedad ofrece a la labor de rastrado la diferencia que hubo entre la máxima y mínima resistencia fue de $130,09 \mathrm{Kg}$, es decir un porcentaje de $24,48 \%$.

En la relación de la resistencia del suelo al rastrado y los grados de humedad de este, se observa que el tratamiento testigo es el que mayor resistencia ofreció al rastrado, descendiendo bruscamente en el tratamiento A1R1 con 14,59\% de humedad y manteniéndose casi constante hasta el tratamiento A8R8 con 30,59\% de humedad. El tratamiento que menor resistencia ofreció fue el A7R7 con 27,98\% de humedad. En el tratamiento A9R9 la resistencia asciende bruscamente. En los tratamientos con $30,59 \%$ y $30,75 \%$ de humedad se vuelve a observar que el suelo se adhiere a los discos de la rastra.

Una de las razones por la que en los extremos la resistencia sea mayor que en la labor de rastrado es porque en los suelos muy secos la rastra hace mayor esfuerzo al romper mayor número de terrones y de mayor diámetro, y en los suelos muy húmedos se toma en cuenta la compactación del suelo por parte de los neumáticos del tractor que es significativa y además que el suelo se encuentra 
muy pesado. La otra razón es que la rastra tiene mayor contacto con el suelo por el número de discos que posee y porque su peso es mayor.

En cuanto al tiempo empleado en la labor de arado los cuatro primeros tratamientos presentaron los mayores tiempos, siendo el tratamiento A3R3 con 18,65\% de humedad el que mayor tiempo registro con 75 segundos por cada pase, que llevado a hectárea es de $3 \mathrm{H} 28 \mathrm{~m} 20$ s de tiempo efectivo empleado en la labor, y el tratamiento de menor tiempo fue el de A8R8 (30,59\% de humedad) con 56,44 segundos por cada pase que llevado a hectárea representa $2 \mathrm{H} 36 \mathrm{~m} 47 \mathrm{~s}$ de tiempo efectivo. Comparando estos dos valores la reducción del tiempo fue de 24,75\%. Es importante señalar que el tiempo más bajo registrado en el tratamiento A8R8 se debe a que como el suelo se adhería al implemento, este realizaba la labor solo superficialmente.

Realizando un estudio en el tiempo empleado en la rastrada, al igual que en el arado, los primeros cuatro tratamientos registraron los mayores tiempos, siendo el tratamiento A1R1 (14,05\% de humedad), el que mayor tiempo registro con 51,67 segundos, que llevado a hectárea representa $1 \mathrm{H} 35 \mathrm{~m} 41 \mathrm{~s}$, y el tiempo menor registrado fue en el tratamiento con 25,28\% de humedad (A6R6) con 37,67 segundos en cada pase lo que representa un tiempo efectivo de $1 \mathrm{H} 09 \mathrm{~m} 46 \mathrm{~s}$ por hectárea en realizar la labor. Comparando ambos valores, la reducción del tiempo fue de 27,10\%.

Comparando los tiempos del arado con relación a los del rastrado, los primeros son superiores en todos los tratamientos.

Con relación al grado de pulverización del terreno, tanto como en el arado como en el rastrado los suelos secos (con 14\% de humedad como en la parcela testigo) son los que peor quedan trabajados basado en el estudio del diámetro y número de terrenos, en los tratamientos con suelo húmedo son los que mejor quedan preparados, pero surge el inconveniente de que presentan mayor compactación y el suelo se adhiere a las rejas y discos de los implementos.

Con relación al diámetro de los terrones se redujo en un 20,03\% en el arado y de $13 \%$ en el rastrado. Sin embargo, la reducción más significativa se dio en el número de terrones donde se redujo en un $52,92 \%$ en el arado y en $64,17 \%$ en el rastrado, según los gráficos 5 y 6 realizados para el efecto.

\section{Conclusiones}

- El grado de humedad existente en un suelo franco limoso influye en las labores de arado y rastrado, sobre todo en la resistencia que ofrece al suelo las labores, así como en el grado de pulverización del terreno. 
- Las máximas resistencias registradas se dieron al inicio de las operaciones.

- En un suelo franco limoso entre el 14\% y el 19\% de humedad no es recomendable realizar las labores primarias, tanto por la resistencia que ofrece el suelo, como el tiempo empleado y el grado de pulverización del terreno, lo que a la postre afecta al rendimiento de la maquinaria e incide en lo económico.

- En un suelo franco limoso con un grado de humedad superior al $28 \%$ no es recomendable realizar las labores de arado y rastrado ya que el suelo se adhiere a las rejas y discos de los implementos, por la compactación que está expuesto el mismo y porque la resistencia aumenta en estos grados de humedad.

- En un suelo franco limoso arado con un grado de humedad recomendable, entre los valores máximos y mínimos estudiados puede disminuir hasta un 37\% la resistencia; un 24,75\% el tiempo empleado; un 20,03\% el diámetro de los terrones y en un 52,92\% en número de terrones.

- Un suelo franco limos rastrado con un grado de humedad recomendable, entre los valores máximos y mínimos estudiados puede disminuir hasta en un 24,48\% la resistencia; un 27,10\% el tiempo empleado; un 13,00\% el diámetro de los terrones y en un 64,17\% el número de terrones.

- La importancia de la labor del rastrado en la pulverización del terreno es notoria, al disminuir considerablemente el número y diámetro de terrones.

- En todos los factores estudiados, la disminución del porcentaje entre el máximo y mínimo valor registrado fue significativo.

- Se determinó que es conveniente realizar las labores de arado y rastrado entre el 20\% y el $27 \%$ de humedad.

- Finalmente, cuando se realizan las labores citadas en un suelo seco, no queda convenientemente preparado, y en un suelo demasiado húmedo, se nota la compactación que sufre el suelo a más que este se adhiere a los implementos. En ambos casos se registraron las resistencias más altas.

\section{Referencias Bibliográficas}

Arnal, P y Laguna Blanca, A. (2006). Tractores y motores agrícolas: Ministerio de Agricultura, Pesca y Alimentación, Madrid, España. $6^{\circ}$ edición. 
Buduba, Carlos (2004). Muestreo de suelos; criterios Básicos. Revista Patagonia Forestal Año X No 1. Laboratorio de suelos CIEFAP-UNPSJB.

Castilla, F. (2013). Siembra Directa. La elegida para conservar el suelo: Una decisión agronómica que combina rotación de cultivos, fertilizantes y agricultura de precisión para aumentar la producción y preservar los recursos naturales. Revista de Investigación Agropecuaria., vol. 39, núm. 2, pp. 118-123.

Fernández Gálvez J.; Simmonds, 1. P. y Barahona, E., (2005). Interpretation of soil moisture profiles on gravel rich soils derived from soil dielectric measurements. En: Sustainable Use and Management of Soils -Arid and Semiarid Regions-, Advances in GeoEcology 36, 241250. Eds. A. Faz Cano, R.

Gavande Samp.at A (1991). Física de suelos. Principios y Aplicaciones. Editorial Limusa. México.

Gill, N y Van denBerg, J. (1998). Retos y oportunidades en la ciencia del suelo - Sociedad Española: Recuperado en: www.secs.com.es/wp-content/.../Retos-y-Oportunidades-en-lasCiencias-del-Suelo.

González, G. (2004). Métodos estadísticos y principios de diseño experimental. Universidad Central del Ecuador. Quito.

Hybel, D. (2006). Cambios en el complejo productivo de maquinarias agrícolas 1992-2004. Instituto Nacional de Tecnología Industrial, Economía Industrial. Argentina Keen, M y Maíz, G (1925). Diseño y construccion de un dinamómetro para la medición. Recuperado en: https://dialnet.unirioja.es/descarga/articulo/4838409.

Mehuys, G; Tiessen K; Villatoro M; Sancho F y Lobb, D. (2009). Erosión por labranza con arado de disco en suelos volcánicos de ladera en Costa Rica. Revista Agronómica Costarricense., vol. 33, núm. 2, pp. 205-215.

Nichols, R. (1929). Research genealogy. Recuperado en: https://www.ancestry.com/genealogy/.../frank-nichols_12099058.

Putte, G; Govers, J; Diels, C; Langhans, W; Clymans, E; Vanuytrecht, R y Merckx D. (2012). Soil functioning and conservation tillage in the Belgian Loam Belt, Soil and Tillage Research, vol. 122, no 0 , pp. 1-11, 2012.

Ribes, J y Marcos N. (2005). Estudios de compactación de suelos: neumáticos y tractores. Vida Rural, pp. 48-52. 
Rothanested, S (2015). Resistencia de materiales- Recuperado en: https://rephip.unr.edu.ar/.../20401-15\%20Resistencia\%20DE\%20Materiales...

Zagal, Erick (2007). Protocolo de métodos de análisis de suelo y lodos. Universidad de Concepción. Faculta bajamente de agronomia. 